\title{
Low-background, digital gamma-ray spectrometer with BEGe detector and active shield: commissioning, optimisation and software development
}

\author{
Krzysztof Gorzkiewicz $^{1}$ (i) $\cdot$ Jerzy Wojciech Mietelski ${ }^{1} \cdot$ Renata Kierepko $^{1} \cdot$ Kamil Brudecki $^{1}$
}

Received: 24 July 2019 / Published online: 18 October 2019

(c) The Author(s) 2019

\begin{abstract}
This paper presents results of the development process of low-background, digital gamma-rays spectrometer equipped with Broad Energy Germanium detector (CANBERRA BE5030), multi-layer passive shield and cosmic veto system that consists of five plastic scintillators (SCIONIX EJ-200). Data acquisition is performed using digitizer CAEN DT5725 with CoMPASS software. Output data analysis is carried out with purposely written and developed code VETO. On the basis of conducted tests, acquisition parameters were set up and tuned as well as time delays between all detectors were established. As a result of the configuration process, the mean background counts reduction of $64 \%$ in the whole spectrum and $65 \%$ in annihilation peak were achieved. This procedure allowed diminishing detection limits of selected isotopes $37 \%$ on average.
\end{abstract}

Keywords Low-background measurements $\cdot$ Low activity $\cdot$ Digital gamma-ray spectrometry $\cdot$ BEGe $\cdot$ Cosmic muons veto detector $\cdot$ Plastic scintillators

\section{Introduction}

Gamma-ray spectrometry is one of the most versatile and commonly used nuclear radiation spectrometric technique. It finds applications both in qualitative and quantitative analyses of gamma-ray emitting isotopes. Its comprehensive character allows using this method in measurements of both high-radioactive research materials as well as those containing a concentration of radioisotopes at the trace level like environmental samples [1-3]. However, in the latter case, the key point is to achieve accordingly low detection limits. It is possible under certain conditions and one has to meet a few requirements namely: utilize proper germanium detectors with high efficiency of gamma-ray absorption as well as advanced shielding systems (passive and active) allowing to reduce gamma radiation spectrometer background. Passive system is implemented by both appropriate selection of materials surrounding the detector and the introduction of radiopure nitrogen gas to eliminate radon progeny whereas

Krzysztof Gorzkiewicz

krzysztof.gorzkiewicz@ifj.edu.pl

1 Institute of Nuclear Physics, Polish Academy of Sciences, Radzikowskiego 152, 31-342 Kraków, Poland active option based on additional detectors (Charpak chamber or plastic scintillator) for an anticoincidence set-up. Its purpose is to reduce detector background induced by nuclear interaction of cosmic rays with passive shieldings and germanium detector [4-6].

The most important components of gamma-rays background are terrestrial external $\gamma$ radiation emitted by naturally occurring radionuclides, internal gamma radiation from radionuclides present in traces in construction materials of detector and shield, neutron background due to secondary cosmic radiation and produced in spontaneous fission of $\mathrm{U}$, Th as well as e.g. $(\alpha, n)$ reactions. The last but not least are also secondary cosmic ray muons that may directly deposit energy in germanium crystal as well as produce additional neutrons in several processes: muons capture, muon-induced spallation reactions, muon-induced hadronic and electromagnetic cascades $[1,7,8]$.

From the low background measurements point of view, quite remarkable is recent progress in spectroscopic electronics and digital signals processing systems, allowing to replace few discrete electronic devices creating gamma-rays spectrometer (like shaping amplifier, Analogue to Digital converter (ADC), logic units) with one, fast, compact multiinputs digital analyzer (digitizer) that provides information about time, energy and pulse shape. As a result, reduction 
of a several single-purpose components is possible what along with the conversion of an analogue signal into digital form as the first step of signal processing, improvements in spectrometer parameters like energy resolution are achievable [9].

In this paper, we present the construction and commissioning process of low-background, digital gamma-ray spectrometer with active shield (cosmic muons veto system) and digital analyser as an acquisition and signal processing device. The spectrometer is located in a ground level laboratory in the Department of Nuclear Physical Chemistry Institute of Nuclear Physics Polish Academy of Sciences (IFJ PAN), Krakow, Poland. Additionally, the paper includes results of tuning and optimisation processes of the most critical digitizer parameters as well as a description of custom software named VETO used for off-line analysis of data generated by the digitizer. Finally, the results of measurements carried out using the described gamma-ray spectrometry system are presented.

\section{Experimental}

\section{Germanium detector}

Presented gamma-ray spectrometer consists of Broad Energy Germanium (BEGe) detector BE5030 with relative efficiency $\geq 48 \%, 0.6 \mathrm{~mm}$ Carbon Composite window with $102 \mathrm{~mm}$ diameter of endcap in U-Style standard cryostat manufactured by Canberra which offers both high efficiency and good energy resolution (FWHM $2.0 \mathrm{keV}$ for $1332 \mathrm{keV}$ energy line). The detector is supplied with $3.5 \mathrm{kV}$ high voltage (Canberra model 3106D).

\section{Passive shield}

Composite passive shield of the spectrometer is in cubic shape and consist of several layers (from outside to inside): 8 to $12 \mathrm{~cm}$ of paraffin (neutron moderator), $5 \mathrm{~cm}$ of plastic scintillator (part of active shield), $10 \mathrm{~cm}$ of standard lead (to attenuate $\gamma$-rays, activity $<2000 \mathrm{~Bq} / \mathrm{kg}$ of ${ }^{210} \mathrm{~Pb}$ ), $2 \mathrm{~mm}$ of cadmium (to absorb the thermal neutrons), $5 \mathrm{~cm}$ of old lead (over 2500 years from casting, low ${ }^{210} \mathrm{~Pb}$ content, about $6-8 \mathrm{~Bq} / \mathrm{kg}$, its role is to attenuate gamma-radiation emitted after neutron capture in $\mathrm{Cd}$ ), $1 \mathrm{~cm}$ of electrolytic copper (to attenuate lead fluorescent X-rays) and 0.5 to $1 \mathrm{~cm}$ of acrylic organic glass (low energy X-rays absorbent). The passive part of the shield was constructed in 1998 and now the set of plastic scintillators replaced previously used Charpak chamber localized only at the top of the shield [10]. Additionally, flush of vapours of liquid nitrogen from Dewar purge spectrometer chamber in order to diminish the concentration of radon progeny e.g. ${ }^{214} \mathrm{~Pb},{ }^{214} \mathrm{Bi},{ }^{212} \mathrm{~Pb}[11]$. The volume of internal spectrometer chamber is about $16,5 \mathrm{dm}^{3}$.

\section{Active shield}

The active shieldsetup consists of five large area, $5 \mathrm{~cm}$ thick, plastic scintillation detectors type EJ-200 with build-in HV supply manufactured by SCIONIX [12]. They are mounted to the lead walls surrounding germanium detector and play a role of additional active shielding allowing to reduce spectrometer background induced by the interaction of cosmic rays (mainly muons but also nucleons) with detector materials and passive shield [4, 5]. Two of the scintillators are placed horizontally above and below BEGe detector: TOP (number 2, area $70 \mathrm{~cm} \times 70 \mathrm{~cm}$ ) and BOTTOM (number $3,30 \mathrm{~cm} \times 50 \mathrm{~cm}$ ). Rest of them are situated vertically: FRONT (number 4, $30 \mathrm{~cm} \times 50 \mathrm{~cm}$ ), SIDE (number 5, $30 \mathrm{~cm} \times 50 \mathrm{~cm}$ ) and REAR (number $1,30 \mathrm{~cm} \times 45 \mathrm{~cm}$ ). Relative positions of all detectors are presented in Fig. 1.

\section{Acquisition setup}

Acquisition of the experimental data is performed by digitizer CAEN DT5725 [13]. Main features of such digital analyser are: 8 detectors inputs with the simultaneous acquisition (in our setup 6 are occupied-1 by BEGe detector and 5 by scintillators), 14 bits flash ADCs with sampling rate $250 \mathrm{MS} / \mathrm{s}$ for each channel and USB connection to PC. Data, registered with a time resolution up to $4 \mathrm{~ns}$, consist of information about time stamps and energy

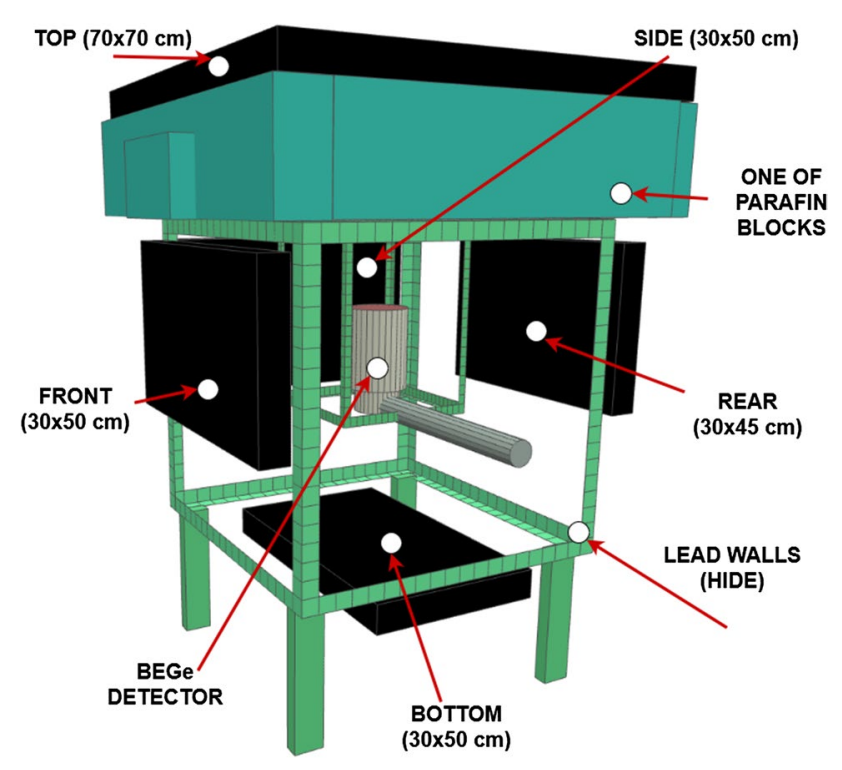

Fig. 1 Relative position of detectors of low-background, digital gamma-ray spectrometer used in the Institute of Nuclear Physics Polish Academy of Sciences. Some elements of passive shield are hidden 
of incoming pulses (additionally code of error is saved as a third digit). Also, waveforms visualisation and acquisition is possible what is useful during the digitizer configuration process [13].

Readout chain is fully digital due to the fact that analogue pulses from preamplifiers are converted into digital form by fast flash ADCs at the entry of the device. Then, a digital pulse is processed by algorithms based on the utilization of timing and trigger filter RC-CR ${ }^{2}$ and Trapezoidal filter [13-15]. The first one performs smoothing ( $\mathrm{RC}-$ low-pass filter), removes low frequency noises and transforms the signal from preamplifier into bipolar pulse, whose zero crossing is used in the determination of pulse time stamps. The latter one, Trapezoidal filter, transforms the signal from preamplifier into a trapezoid shape with the flat top region, which height is proportional to input pulse amplitude (corresponding to the energy deposited in the detector).

The great advantage of such approach, in contrary to analogue systems which consist of separated, designed for particular purpose electronic instruments (i.e. amplifiers, ADC, logic units), is that digitizers are multi-purpose systems and all necessary elements are packed in one compact device.

However, proper tune of spectrometer operating parameters (total over 50 options that must be set up prior measurements) and their values optimisation process, which takes into account the character of the planned research, requires knowledge in Digital Signal Processing and is crucial in order to achieve the best quality of results (detector timing synchronization and energy resolution) [9]. Control of the analyser is performed with dedicated readout software provided by the manufacturer-CAEN CoMPASS Multiparametric DAQ Software for Physics Applications [9].

The most important of those parameters are: number of signal samples used in input pulse baseline calculations (called in CoMPASS software Ns baseline), signal threshold values (Threshold), rise times of trapezoidal and $\mathrm{RC}-\mathrm{CR}^{2}$ filters (Trapezoid Rise Time and Input Rise Time), trapezoid flat top width (Trapezoid Flat Top), number of signal samples in Flat Top region used for energy calculations ( $N s$ Peak) and minimum time between two trapezoids (Peak Hold off) [9].

The determined operating parameters are saved as a configuration file. This approach allows to reduce the number of manual regulations, ensures reliability and repeatability. One of the most important advantages is building a library for fast and easy tailoring of spectrometer parameters for different kinds of experiments.

The fully digital systems, in comparison with analogue ones, produce a high amount of data (even tens of gigabytes, depending mainly on signal threshold values for given detector and time of measurement) that must be stored and processed to obtain desired measurements information (e.g. energetic spectra). However, this approach allows using collected data repeatedly in various analyses as well as in multiple experiments.

As a result of conducted measurements, 6 output files (in $\{* . c s v\}$ format) are generated. They consist of information of energy as well as time stamps of incoming pulses for each of detectors separately. Those raw data must be further analysed by purposely written software e.g. VETO code.

\section{Off-line analysis software: VETO}

Further off-line analyses of raw output files generated by digitizer DT5725 are performed utilizing VETO code. It is an application written in $\mathrm{C}++$ programming language with graphical user interface (prepared with WxWidgets 3 library [16]). This code has two primary parts-BASIC and ADVANCED. The first one is used for the fastest and user-friendly preparation of anticoincidence spectra. The impulses recorded by the BEGe detector (their timestamps and energy) for which the coincidence phenomenon did not occur are saved in a separate file, otherwise record is rejected. Also, it creates a file in $\{*$.tka $\}$ format containing an anticoincidence energy spectrum. This part of the software is intended to use in routine measurements conducted by means of low-background spectrometer.

The ADVANCED part of the software allows also to test coincidence events between the freely chosen subset of detectors (since the analyses are performed off-line, any desired logical functions between signals (prompt or delayed) can be executed), determine the delays between detectors, create sequential spectra (divide one output files into many others, arranged in time, with specific length) and quickly create energy spectra or sum up spectra. The code is also prepared to deal with CAEN $\mathrm{MC}^{2}$ output files-previously used to control CAEN digitizers.

\section{Results and discussion}

\section{Configuration and optimization process}

As it was mentioned in the experimental part, prior first measurements, it is necessary to conduct tuning and optimization of the spectrometer operating parameters. In this paper, the results of the tuning process of following working parameters of digital filters implemented into digitizer: Trapezoid Rise Time, Ns Baseline and Ns peak are presented. Those parameters have, among others, crucial influence on final energy resolution of spectra obtained by BEGe detector. Conducted experiments consisted of measuring of FWHM of selected peaks as a function of output count rate (OCR, which in our experiments, is equal to Input Count Rate [17]).

Figure 2 presents width of ${ }^{137} \mathrm{Cs}$ energy line of $662 \mathrm{keV}$ (Gaussian fit curve width $\sigma, \mathrm{FWHM} \approx 2.35 \sigma$ ) as a function 
Fig. 2 The relationship between FWHM of ${ }^{137} \mathrm{Cs}$ energy line of $662 \mathrm{keV}$ and the Trapezoid Rise Time values obtained for a lowbackground digital gamma-ray spectrometer located at the IFJ PAN. In case of low count rates, the best results are achieved for higher values of Trapezoid Rise Time (dashed line ellipse). In contrary, for higher count rates (above $30 \mathrm{~Hz}$ ), the user should choose lower values of Trapezoid Rise Time (solid line ellipse)

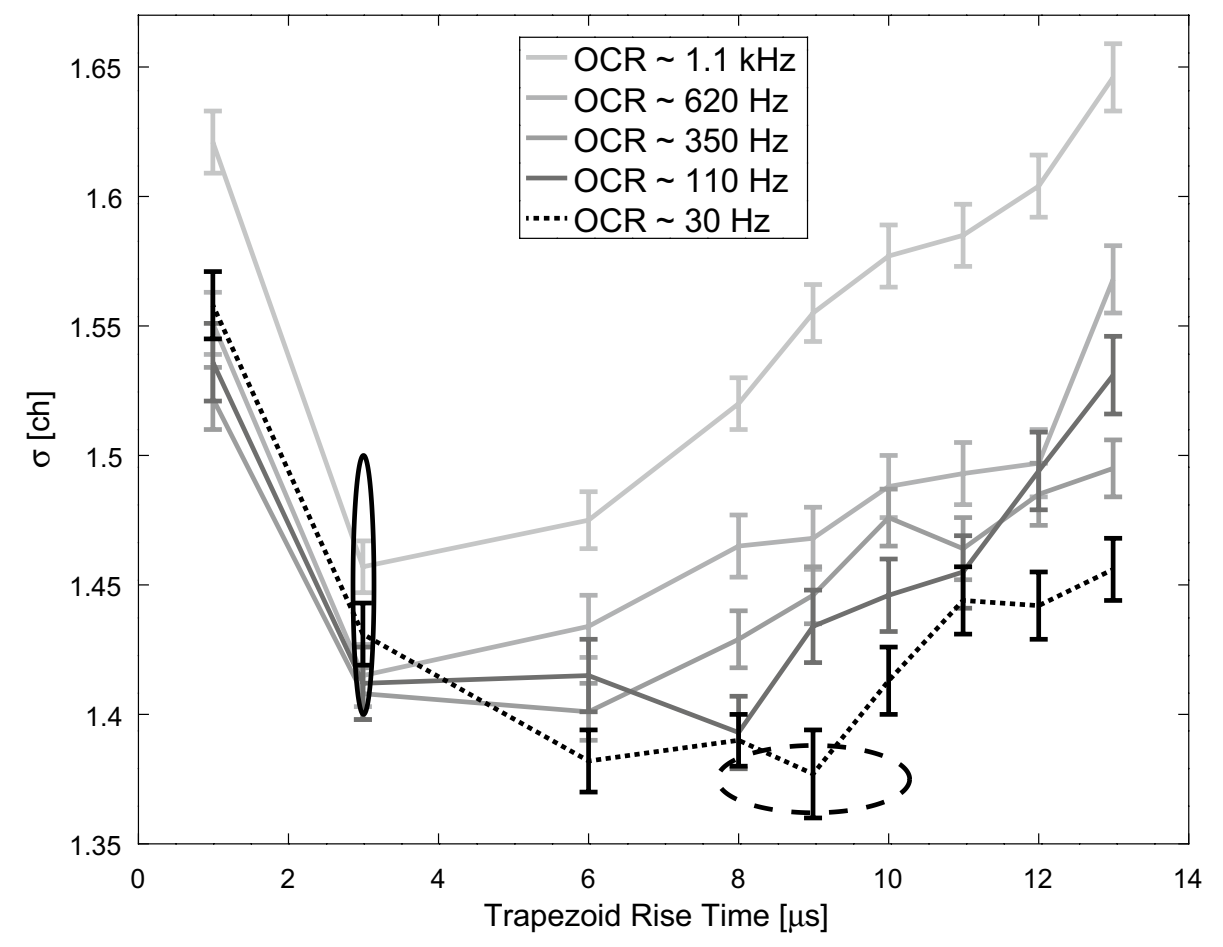

of Trapezoid Rise Time values (which corresponds to analogue shaping time [9]) for various OCRs.

The experiment shows that along with decreasing count rates values, one should set higher values of Trapezoid Rise Time. Taking into account that spectrometer is designed for trace radioactivity measurements (low OCR, dotted line), the best result was obtained for value $8.992 \mu$ s (dashed line ellipse). In the case of experiments that involve measurements of samples with higher count rates (above $30 \mathrm{~Hz}$ ), the user should choose lower values of Trapezoid Rise Time (solid line ellipse).

Next, energy resolution against Ns Peak values was examined (Fig. 3). The best energy resolution was achieved for the lowest value of Ns Peak equal one signal sample (dashed line ellipse).

Finally, experiments show that in case of low values of OCRs, one should choose as high number of signal samples used in input pulse baseline calculations as possible (Fig. 4). In our case, it was 16,384 samples (dashed line ellipse). However, along with increasing OCRs (from $1.1 \mathrm{kHz}$ ), one should take lower values of Ns Baseline in order to keep the desired energy resolution (for the highest value of Ns Baseline, significant increase in peak width is observed-solid line ellipse). This phenomena is caused due to shorter average time interval between following pulses.

To sum up the optimization process of the digital gamma ray spectrometer, user should very carefully choose acquisition parameters in order to obtain the best results in planned experiments taking into consideration features of measurements e.g. average count rates.

\section{Detectors mutual delays}

Utilizing collected spectra and VETO code, we determined the time distribution of coincidences among detectors of our spectrometer and on this basis, their mutual delays. Delays are calculated by subtracting timestamps of chosen detectors. Accordingly to expectations, time differences between scintillation detectors are on the level of up to about $100 \mathrm{~ns}$ while delays between BEGe detector and scintillators are much larger, namely in the range $1.62-1.72 \mu \mathrm{s}$. Figure 5 presents time distributions of coincidences of signal from REAR scintillator and other detectors. All delays have negative values what means that detector REAR registers signals faster than the others (for coincidence events, it has smaller time stamps). The shape of the distribution of coincidences between scintillation and germanium detectors, namely its right-side asymmetry, indicates the presence of both prompt coincidences as well as delayed ones [18].

Determined values of delays may be subsequently saved in the VETO configuration file and utilized in further analyses, for example, during creating anticoincidence spectra. Delays between BEGe detector and scintillators were determined as a point, where the time distribution of coincidences reaches its maximum, and the coincidence window was set at $2000 \mathrm{~ns}$ to ensure that all coincidence events are taken into account. 
Fig. 3 The relationship between FWHM of ${ }^{137} \mathrm{Cs}$ energy line of $662 \mathrm{keV}$ and the Ns Peak values obtained for a low-background digital gamma-ray spectrometer located at the IFJ PAN. The best results are achieved for Ns peak equal one signal sample(dashed line ellipse)

Fig. 4 The relationship between FWHM of ${ }^{137} \mathrm{Cs}$ energy line of $662 \mathrm{keV}$ and the Ns baseline values obtained for a lowbackground digital gamma-ray spectrometer located at the IFJ PAN. In case of low count rates $(\sim 30 \mathrm{~Hz})$, the best results are achieved for the highest values of Ns Baseline (16,384 signal samples, dashed line ellipse)
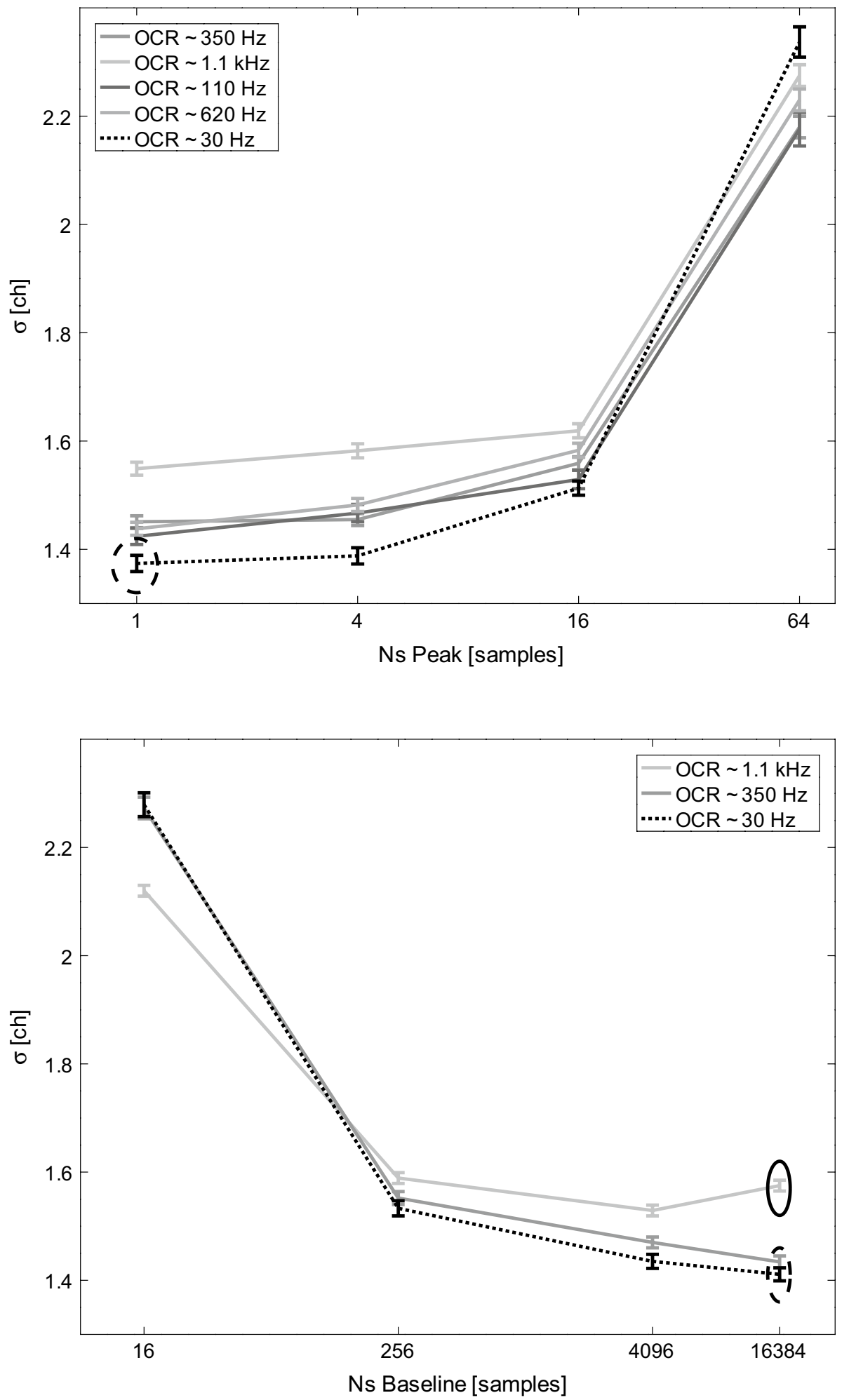

\section{Scintillators threshold values}

As it was mentioned above, the drawback of digital acquisition systems is a huge amount of data generated as a result of single measurement, what may be problematic with data storage, transfer as well as off-line data analyses which may be quite time-consuming. This issue is particularly important for scintillation detectors, which register data with frequency up to several hundreds of $\mathrm{Hz}$ (for a low value of threshold level). Taking into account average time of low-background 
Fig. 5 Time distributions of coincidences of signal from REAR scintillator and other detectors of the lowbackground, digital gamma-ray spectrometer in the IFJ PAN

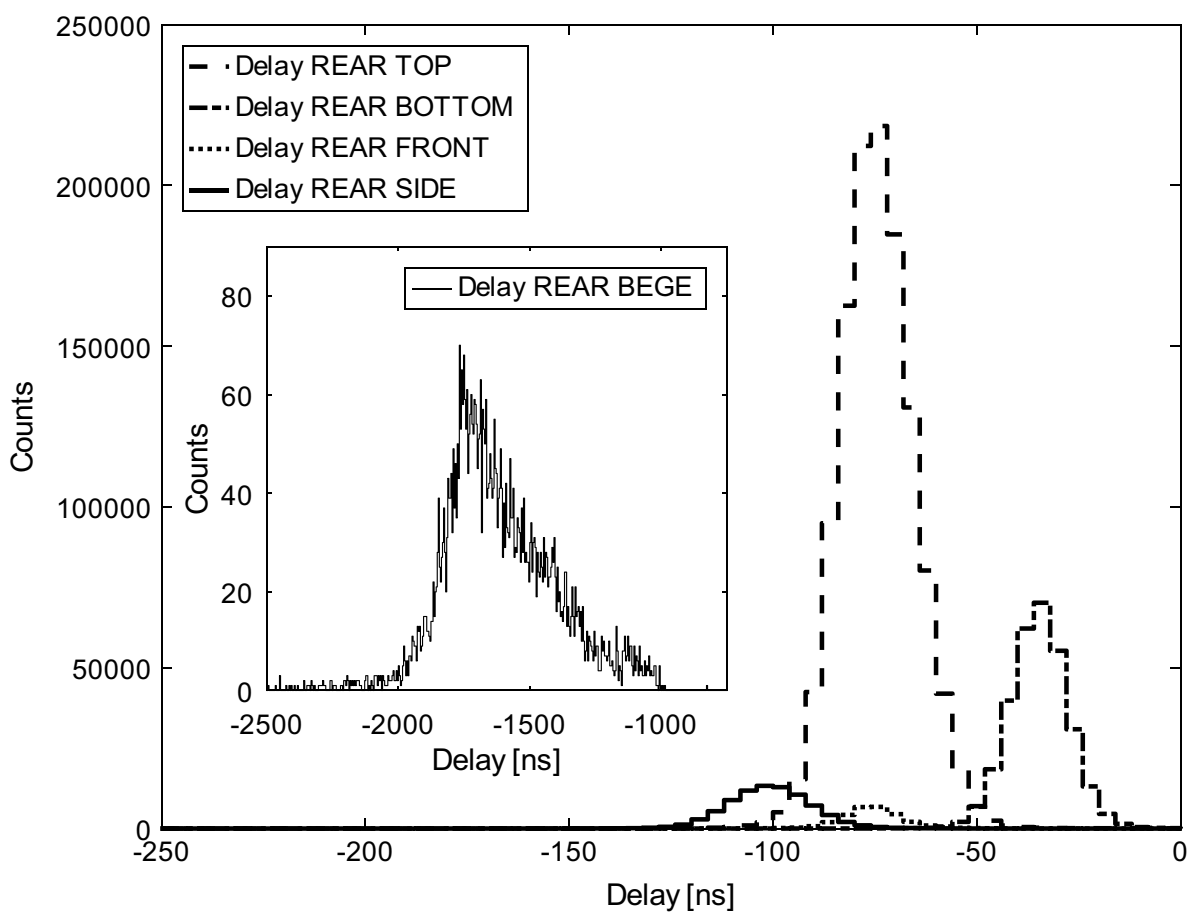

gamma-ray measurements ( 1 week), even $50 \mathrm{~GB}$ of data may be generated.

The spectrum of TOP scintillator is depicted in Fig. 6 (grey solid line). Low-energy part of the spectrum (shaded area between 5th and 400th ADC channels marked with vertical dashed lines) consist mainly of registered gamma radiation with clearly visible Compton edges: $1243 \mathrm{keV}$ and $2381 \mathrm{keV}$ corresponding to gamma-rays emitted by decays of ${ }^{40} \mathrm{~K}(1460 \mathrm{keV})$ and ${ }^{208} \mathrm{Tl}(2614 \mathrm{keV})$, existing in environmental background. The latter spectrum part (above 400th ADC channel) corresponds to muon detection events [19, 20].

To reduce the amount of data register by a spectrometer and then analysed by the VETO code, reduction factors of counts in the whole spectrum of BEGe detector (black squares) and in annihilation line (black circle) were
Fig. 6 Determination of threshold values of scintillation detectorsof the low-background, digital gamma-ray spectrometer in the IFJ PAN. Vertical dashed lines are set to 5th and 400th ADC channels and determine low-energy region of the scintillator spectrum, which consist mainly of registered gamma radiation (shaded area) with marked Compton edges: $1243 \mathrm{keV}$ and $2381 \mathrm{keV}$. The part of the spectrum above 400th ADC channel corresponds to muon detection events. Dotted line draw at 300th ADC channel represents chosen threshold level for scintillation detectors

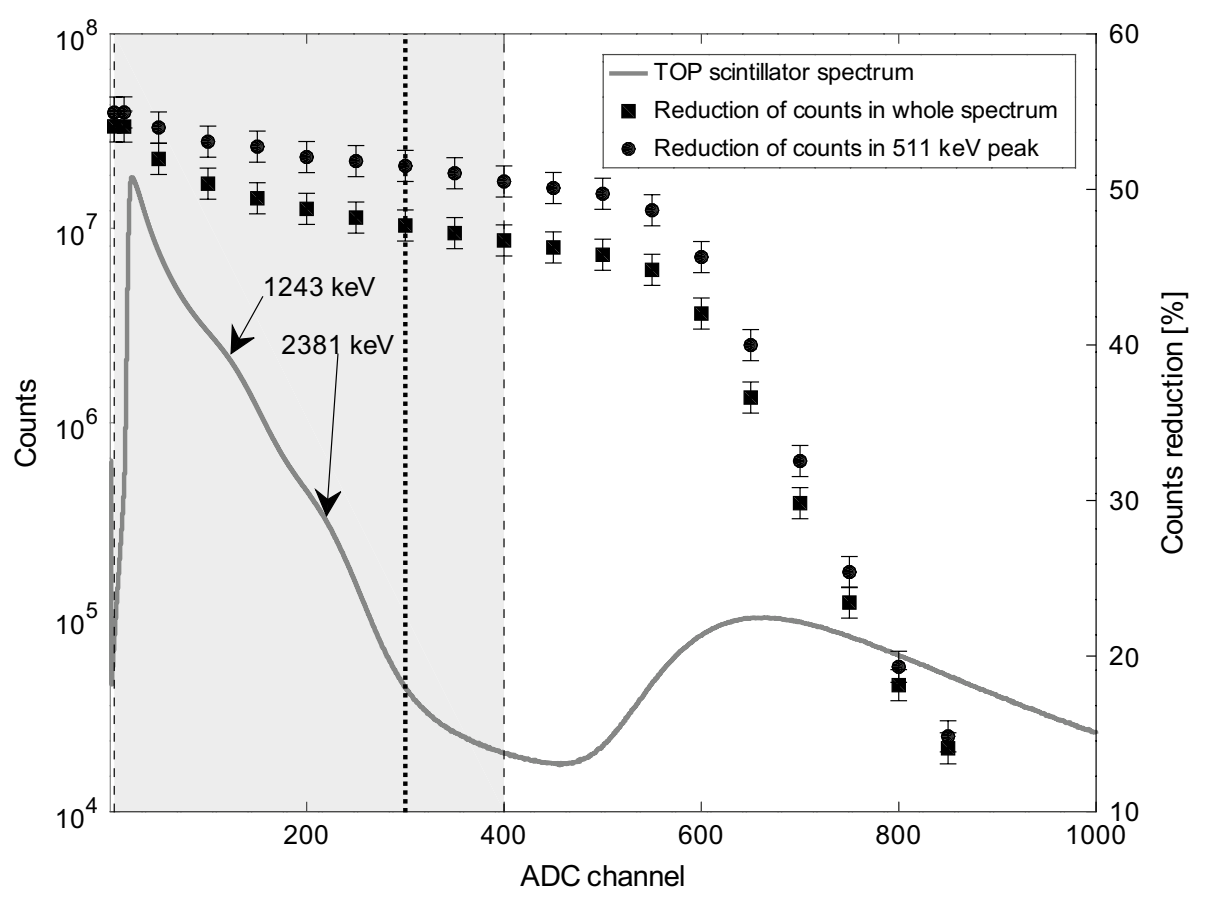


calculated as a function of TOP scintillator threshold level. Examined threshold levels spanned from channel 5th (lowest ADC channel without noise) up to channel 850th (ADC channel above main muon peak). One can see, that gamma radiation registered by the scintillator, did not give an effective increase in background radiation reduction factors. It means that almost all coincidences between scintillation and germanium detectors are due to muon interactions, highlighted by a decrease in reduction factors when the threshold level is above muon peak (located about 650th ADC channel). We decided to set a threshold level for scintillators to 300th ADC channel (in both CoMPASS and VETO software, dotted line). This procedure, allowed to keep high efficiency of BEGe background reduction as well as eliminate a great amount of redundant data. In our setup, we achieved cut of collected data from $7.6 \mathrm{~GB} /$ day $/ 5$ scintillators for threshold level set to 5th $\mathrm{ADC}$ channel down to $310 \mathrm{MB} /$ day/5 scintillators for threshold level set to 300th ADC channel.

Additionally it is worth to mention that during the tests, we do not observe any significant data loss due to deadtime (even with threshold set to channel 5th) what may be explained by application of Multi Event Memory in digitizer, that allows avoiding dead-time between triggers if memory buffers are read and freed faster than they are written. The manufacturer ensures that situation when pulses are rejected due to dead-time will not occur until Input Count Rate is of the order of millions of counts per second [17].

\section{Scintillation detectors spectra}

Figure 7 shows spectra of large area plastic scintillation detectors used in our spectrometer (with previously set threshold level). Two highest curves corresponds to horizontally orientated detectors-TOP and BOTTOM. Three similar, smaller curves represent spectra of vertically situated scintillators mounted to the spectrometer lead walls. It is visible that vertically placed detectors register fewer particles in compare with horizontally positioned ones. This effect occurs due to fact that the distribution of intensity of cosmic ray muons is proportional to $\cos ^{2} \theta$ function, where $\theta$ is a zenith angle of incident particles [21]. Additionally, peaks from vertically oriented scintillators are less pronounced because the area of the detector, seen from muons main direction $(\theta \approx 0)$, is much smaller, therefore, fewer particles hit its surface. Also, the peak is broader for vertically situated detectors, what is an additional manifestation of the angular dependence of the intensity of incoming particles and length of the path travelled in the detector [20].

However, due to their vertical positioning, a small bump at higher energy is present, due to the fact that muons have opportunity to travel longer path and in consequence deposit more energy in the detector [20].

\section{BEGe detector spectrum}

Our spectrometer setup is primarily used in the study of trace radioactivity. This fact implies that the most critical
Fig. 7 Spectra of plastic scintillation detectors utilized in the low-background, digital gamma-ray spectrometer in the IFJ PAN. Curves TOP and BOTTOM corresponds to horizontally orientated detectors, while REAR, SIDE and FRONT curves represent spectra of vertically situated scintillators. Vertically placed detectors register fewer particles in compare with horizontally positioned ones what is cause mainly by the proportionality of the cosmic ray muons intensity to $\cos ^{2} \theta$ function $(\theta$ is a zenith angle of incident particles)

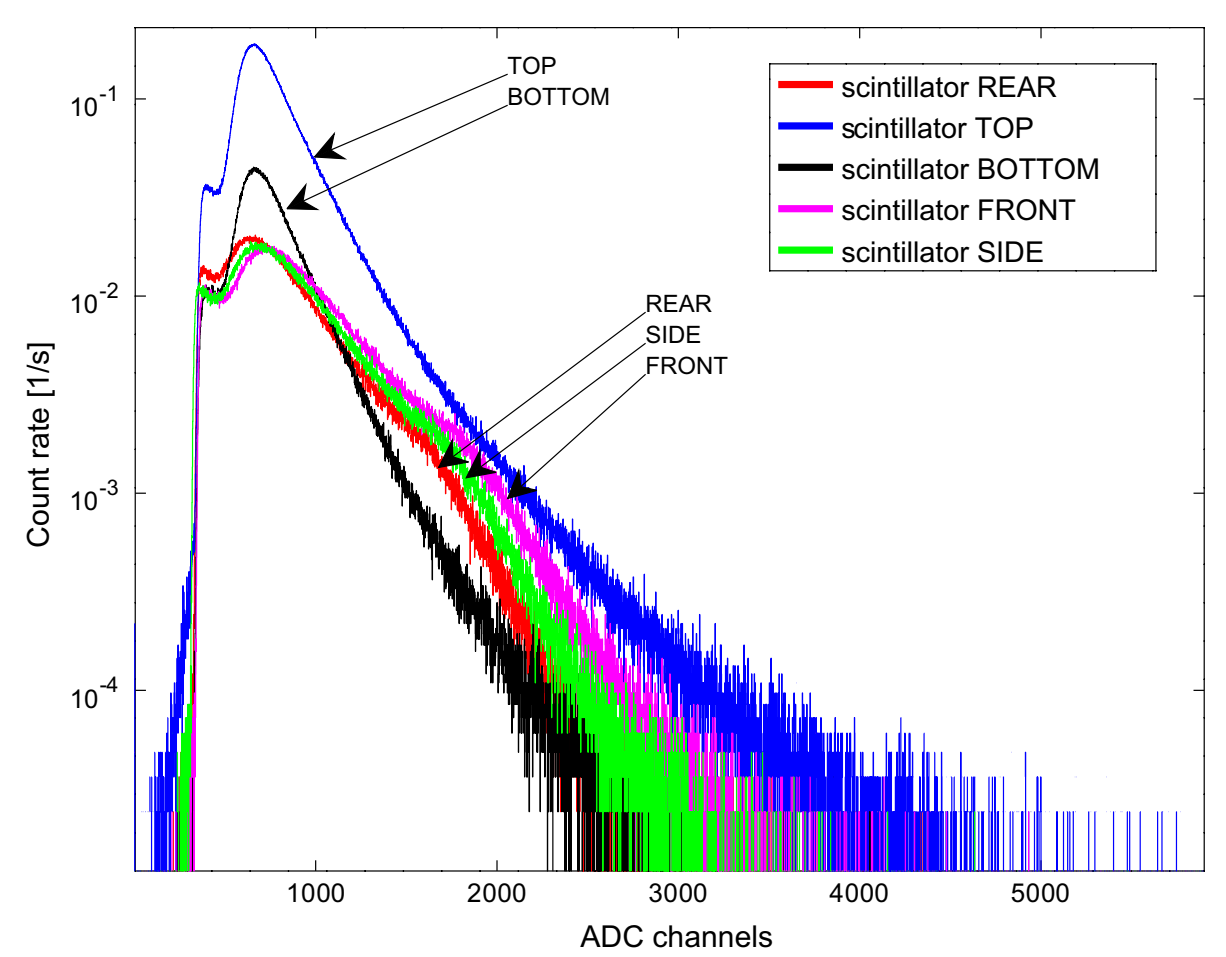


task of configuration process was a reduction of germanium detector background. That was achieved using a multi-layer passive shield, purging of inner spectrometer chamber with liquid nitrogen vapours and cosmic ray veto system. Figure 8 depicts $\gamma$-ray spectra before application of anticoincidence system (VETO OFF, black solid line), with applied liquid nitrogen purging (VETO OFF + LN2, green solid line) and after rejecting of coincidence events (VETO ON $+\mathrm{LN} 2$, blue solid line). The applied setup allowed to achieve reduction background counts of $64 \%$ (from $1.21 \mathrm{cps}$ down to 0.43 cps in energy range $40-2700 \mathrm{keV}$ ). The cosmic veto system contributes in 59\% reduction of total background counts while the radon reduction system caused only $5 \%$ of reduction. Annihilation line that corresponds to pair production processes induced by high-energy gamma-rays and muons interaction [22] was reduced by $65 \%$. Figure 9 shows BEGe detector spectrum in range below $1 \mathrm{MeV}$. Calculated reduction factors of net counts in selected peaks derived from cosmic radiation (along with interactions in spectrometer materials) were presented in Table 1. Lines induced by both inelastic scattering of fast neutrons as well as thermalized neutron capture in germanium crystal [7, 22, 23] were reduced from 22.8 to $40.6 \%$ (energy lines $595.9 \mathrm{keV}$ and $691.0 \mathrm{keV}$ ). Peaks induced by neutron capture process in passive shield materials $(\mathrm{Cu}$ and $\mathrm{Pb}$ isotopes) were diminished by $14.3-47.3 \%$ (energy lines $185.9 \mathrm{keV}, 569.8 \mathrm{keV}$, $962.1 \mathrm{keV}, 1115.5 \mathrm{keV}$ and $2614.6 \mathrm{keV}$ ). Additionally, high efficient reduction of gamma ray peaks of radon daughters was achieved, namely $88.6 \%$ and $90.5 \%$ for ${ }^{214} \mathrm{~Pb}(352 \mathrm{keV})$ and ${ }^{214} \mathrm{Bi}(609 \mathrm{keV})$, respectively. However, due to the susceptibility of plexiglasslining, placed inside detector vicinity, to load with static-electricity, it is essential to remove dust that may stick to lining surface and caused significant increment in germanium detector background.

Finally, improvement in detection limits $L_{\mathrm{d}}$ caused by overall background reduction were calculated using formula $L_{\mathrm{d}}=2.71+4.65 \mathrm{~B}^{1 / 2}$ [2] where B is a total background beneath peak area. Estimated gains in detection limits: $36 \%$ for ${ }^{210} \mathrm{~Pb}, 38 \%$ for ${ }^{137} \mathrm{Cs}$ and ${ }^{40} \mathrm{~K}$.

Table 2 presents the contribution of scintillators in BEGe detector background reduction. According to expectations, the most effective in BEGe detector background reduction are scintillators oriented horizontally: TOP and BOTTOM. They are responsible for $49.6 \%$ and $15.0 \%$ of total background counts reduction, respectively. The proportion of reduction factors (3.30) is reflecting exactly the proportion of those detectors areas (3.27). Detectors placed vertically are about 10 times less efficient than the TOP scintillator. This fact may be explained using the same phenomenon as the shape of their energetic spectra-proportionality of incident muons intensity to $\cos ^{2} \theta$ function and smaller surface area. Furthermore, REAR scintillator reduces only about $3 \%$ of the whole background of the germanium detector because it is placed, due to mechanical issue, farther from spectrometer center than the other scintillation detectors. Values in a row ALL (58.7\% and 65.1\% for whole spectrum
Fig. 8 Spectra of BEGe detector (installed in the lowbackground, digital gamma-ray spectrometer located at the IFJ PAN) before and after application of anticoincidence system and liquid nitrogen purging. Smaller figure shows reduction of annihilation line

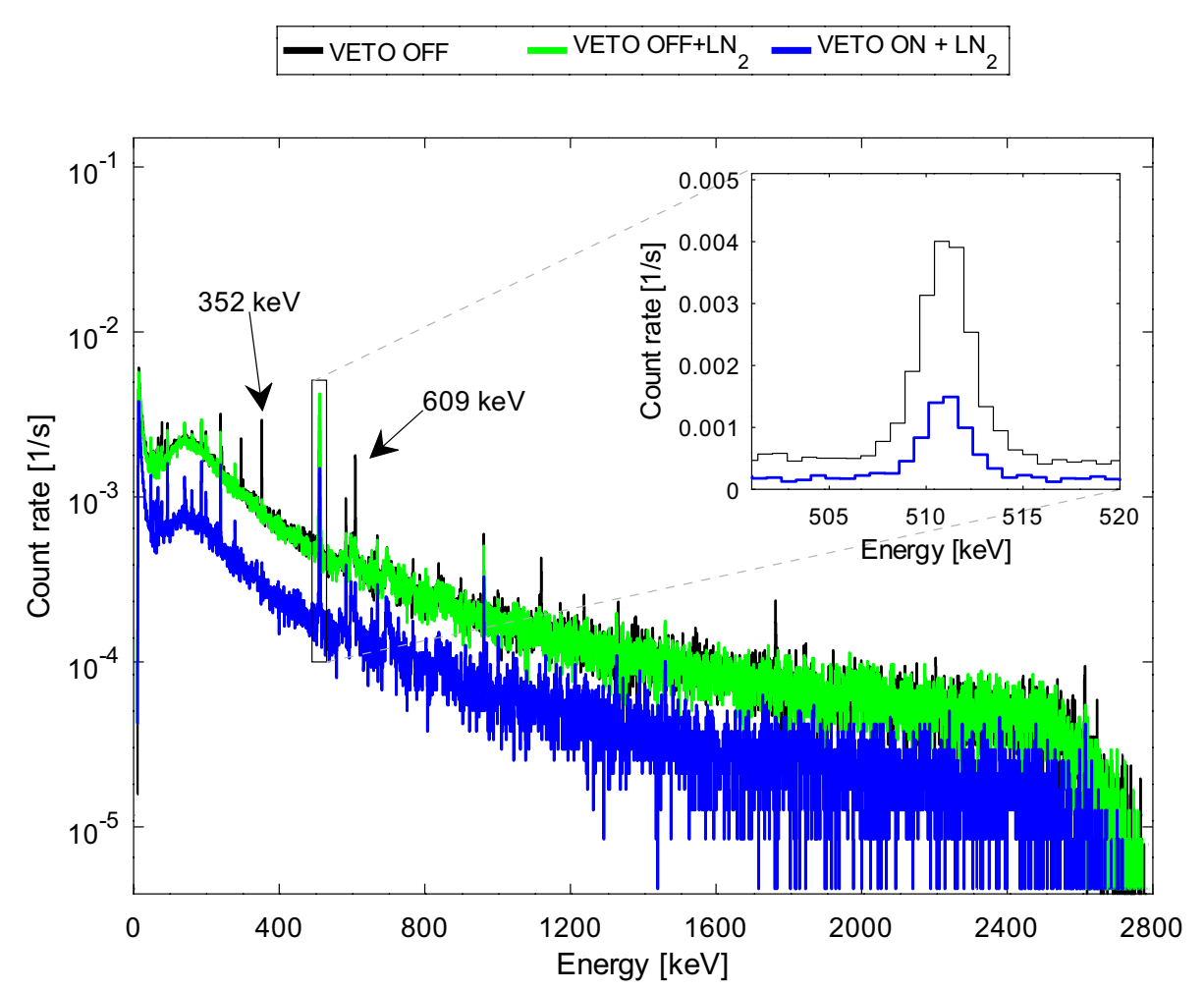


Fig. 9 Spectra of BEGe detector (installed in the lowbackground, digital gamma-ray spectrometer located at the IFJ PAN) in energy range below $1 \mathrm{MeV}$, before and after application of anticoincidence system and liquid nitrogen purging

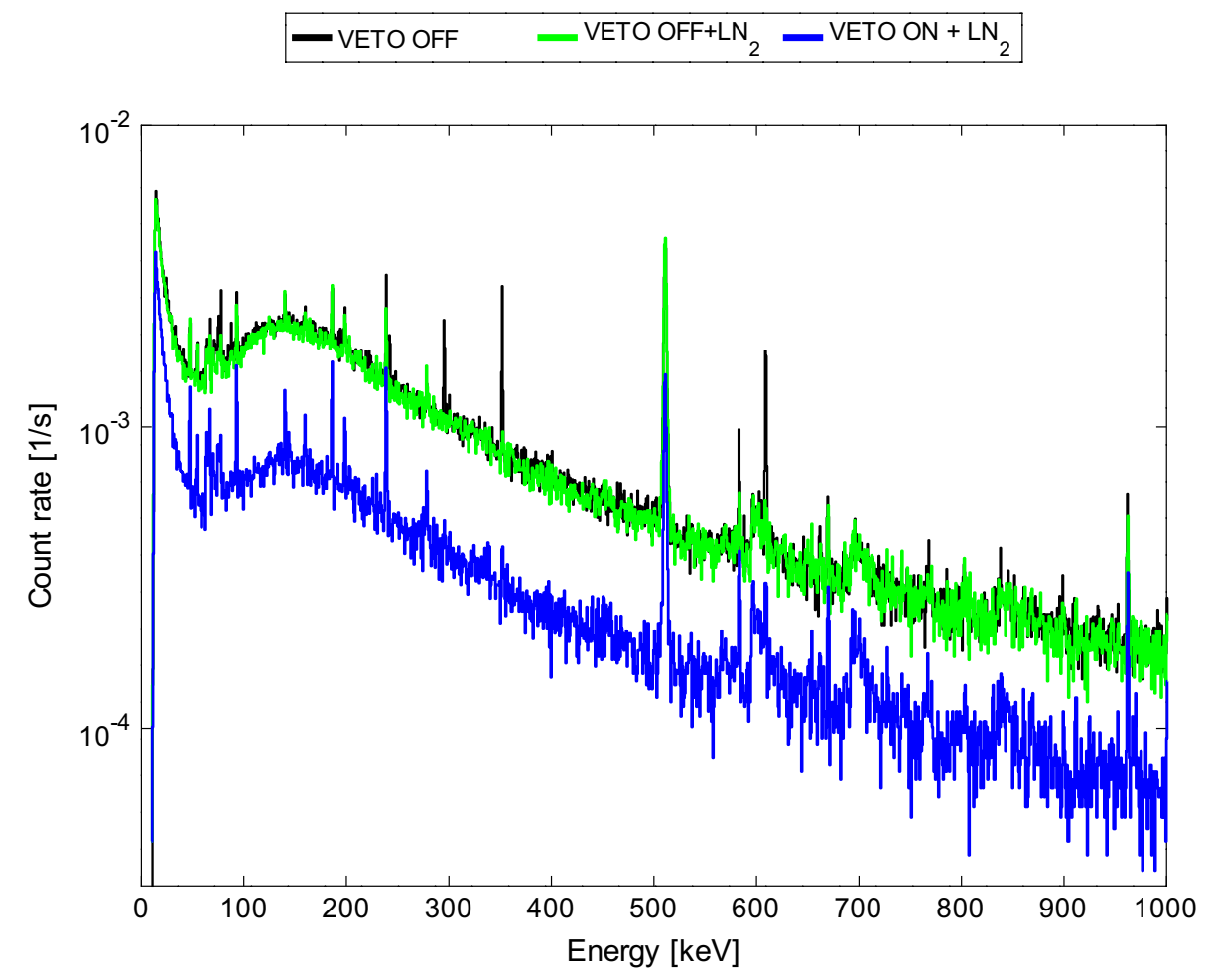

Table 1 Reduction factors of net counts in selected peaks of BEGe detector spectrum, derived from cosmic radiation. The detector is installed in the low-background, digital gamma-ray spectrometer in the IFJ PAN

\begin{tabular}{lll}
\hline Energy $(\mathrm{keV})$ & Reaction/isotope & Reduction $(\%)$ \\
\hline 185.9 & ${ }^{65} \mathrm{Cu}(\mathrm{n}, \gamma){ }^{66} \mathrm{Cu}$ & 14.5 \\
& ${ }^{228} \mathrm{Ac}$ & \\
& ${ }^{22} \mathrm{Ra}$ \\
& ${ }^{235} \mathrm{U}$ & \\
511.0 & Annihilation & 65.1 \\
569.8 & ${ }^{207} \mathrm{~Pb}\left(\mathrm{n}, \mathrm{n}^{\prime}\right)^{207} \mathrm{~Pb}$ & 47.3 \\
& ${ }^{206} \mathrm{~Pb}(\mathrm{n}, \gamma)^{207} \mathrm{~Pb}$ & \\
595.9 & ${ }^{73} \mathrm{Ge}(\mathrm{n}, \gamma)^{74} \mathrm{Ge}$ & 40.6 \\
& ${ }^{74} \mathrm{Ge}\left(\mathrm{n}, \mathrm{n}^{\prime}\right)^{74} \mathrm{Ge}$ & \\
691.0 & ${ }^{72} \mathrm{Ge}\left(\mathrm{n}, \mathrm{n}^{\prime}\right)^{72} \mathrm{Ge}$ & 22.8 \\
962.1 & ${ }^{63} \mathrm{Cu}\left(\mathrm{n}, \mathrm{n}^{\prime}\right)^{63} \mathrm{Cu}$ & 19.8 \\
1115.5 & ${ }^{65} \mathrm{Cu}\left(\mathrm{n}, \mathrm{n}^{\prime}\right)^{65} \mathrm{Cu}$ & 14.3 \\
& ${ }^{65} \mathrm{Cu}\left(\mathrm{p}, \mathrm{n}^{65} \mathrm{Zn}\right.$ & \\
2614.6 & ${ }^{208} \mathrm{Tl}$ \\
& ${ }^{208} \mathrm{~Pb}\left(\mathrm{n}, \mathrm{n}^{\prime}\right)^{208} \mathrm{~Pb}$ & 31.2 \\
\hline
\end{tabular}

and $511 \mathrm{keV}$ peak, respectively, without liquid nitrogen purging), represent reduction factors obtained by the VETO software, which in BASIC part, does not distinguish multiple and single coincidences. The summary contribution of all scintillators in background reduction $(49.6 \%$ (TOP) + $15.0 \%($ BOTTOM $)+6.0 \%($ FRONT $)+5.5 \%($ SIDE $)+2.9 \%$ (REAR)) equals $79 \%$ what, in comparison with observed
Table 2 Contribution of given scintillation detectors in background reduction of the germanium detector installed in the low-background, digital gamma-ray spectrometer located at the IFJ PAN

\begin{tabular}{lcc}
\hline Scintillator & \multicolumn{2}{l}{ Counts reduction $(\%)$} \\
\cline { 2 - 3 } & Whole spectrum & $511 \mathrm{keV}$ peak \\
\hline REAR & 2.9 & 3.7 \\
TOP & 49.6 & 50.9 \\
BOTTOM & 15.0 & 15.5 \\
FRONT & 6.0 & 5.9 \\
SIDE & 5.5 & 6.6 \\
ALL & 58.7 & 65.1 \\
\hline
\end{tabular}

actual reduction efficiency $(58.7 \%)$, means that spectrometer registers $20.3 \%$ multiple events (i.e. registered simultaneously by at least two scintillators).

In order to estimate possible changes in BEGe detector efficiency due to cosmic ray veto system in real samples (air filter after 1 week exposition and 3 reference materials-IAEA 444 [24], IAEA 375 [25] and IAEA 330 [26]), relative differences (with and without active shielding) in net areas of selected peaks were determined (inspired by [27]). Selected research material allowed to perform analysis for wide range of energies, namely from $46.5 \mathrm{keV}$ up to $2.6 \mathrm{MeV}$. Analyses showed that there are no significant changes in detector efficiency what is manifested by the fact that all differences stay close to 0 (in an uncertainty range) what was proved using $t$ test $p$ value $=0.5545$ (Fig. 10). 
Fig. 10 Relative difference in photopeak area for selected energy lines. Anticoincidence system did not introduce any significant changes in net peaks areas. Data collected by means oflow-background, digital gamma-ray spectrometer located at the IFJ PAN

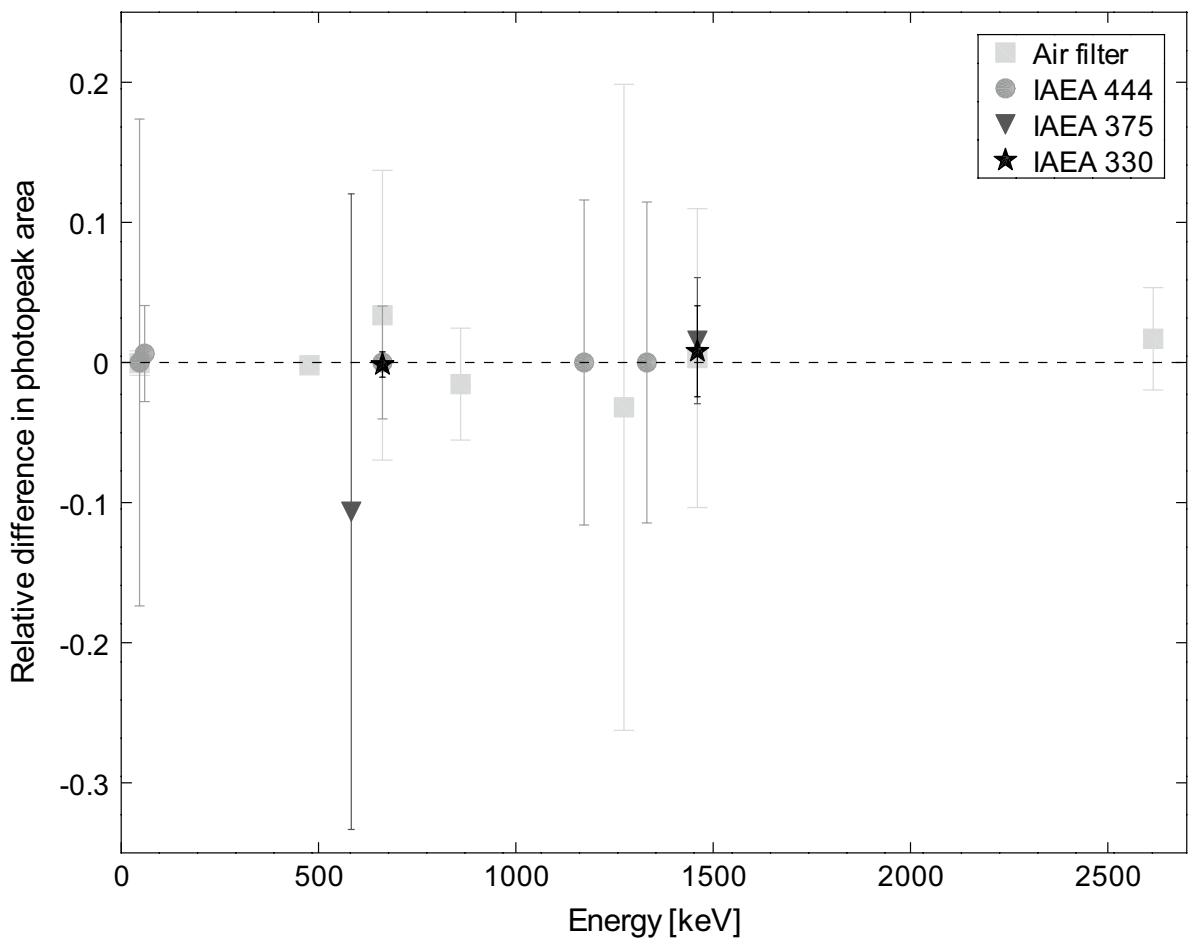

\section{Conclusions}

The configuration and optimization processes of lowlevel,digital gamma ray spectrometer were presented. Spectrometer construction is based on BEGe detector, five large area plastic scintillation detectors, which play a role of an additional, active shield as well as multi-layer passive shield. Data acquisition is done using digitizer CAEN DT5725, and off-line analysis is performed employing purposely written code VETO. It was demonstrated that due to the registration of cosmic ray muons, utilization of multi-layer passive shield and radon reduction system, BEGe detector background was reduced by $64 \%$, while $511 \mathrm{keV}$ peak was diminished over 2.7 times. Additionally, it was proved that the veto system does not deteriorate spectrometer efficiency because it does not reduce net counts in peaks areas. Based on publication of Laubenstein et al. [28], it was estimated that the background of our spectrometer, equipped in anti-cosmic shielding, is comparable with the background of laboratories placed underground, about 20-30 m water equivalent.

Due to the fact, that veto analyses are performed off-line, and unfiltered data from all detectors is stored, it is possible to reuse already collected data in other experiments what is a great advantage, in comparison with traditional, hardware veto systems which do not save all registered data but eliminate anticoincidence events. During offline analyses, digitizer output files (containing time stamps and energies of pulses) are treated as a time series that allow to perform further statistical analyses. In consequence, it is planned to utilize collected data for the investigation of long term cosmic ray muons flux fluctuations.

Acknowledgements This study was partly funded by the National Science Centre, Poland (Grant Number 2018/31/D/NZ7/02217).

Open Access This article is distributed under the terms of the Creative Commons Attribution 4.0 International License (http://creativecommons.org/licenses/by/4.0/), which permits unrestricted use, distribution, and reproduction in any medium, provided you give appropriate credit to the original author(s) and the source, provide a link to the Creative Commons license, and indicate if changes were made.

\section{References}

1. Haines DK, Semkow TM, Khan AJ, Hoffman TJ, Meyer ST, Beach SE (2011) Muon and neutron-induced background in gamma-ray spectrometry. Nucl Instrum Methods Phys Res Sect A Accel Spectrom Detect Assoc Equip. https://doi.org/10.1016/j. nima.2011.01.137

2. Gilmore G, Hemingway JD (1995) Practical gamma-ray spectrometry. Wiley, Chichester

3. Wordel R, Mouchel D, Steinbauer E, Oyrer R (1996) An active background discrimination technique using a multiple detector event by event recording system. Appl Radiat Isot 47:1061-1067

4. Hurtado S, García-León M, García-Tenorio R (2006) Optimized background reduction in low-level gamma-ray spectrometry at a surface laboratory. Appl Radiat Isot. https://doi.org/10.1016/j. apradiso.2006.01.008

5. Heusser G (1993) Background in ionizing radiation detection illustrated by ge-spectrometry. In: Garcia-Leon M, Garcia-Tenorio R, (eds) Proceedings of the 3rd international summer school, 
low-level measurements of radioactivity in the environment, Huelva. World Scientific, Singapore, p 69

6. Trnkowá L, Rulík P (2009) Low background shielding of HPGe detector. Appl Radiat Isot. https://doi.org/10.1016/j.aprad iso.2009.01.079

7. Jovančević N, Krmar M, Mrda D, Slivka J, Bikit I (2010) Neutron induced background gamma activity in low-level Ge-spectroscopy systems. Nucl Instrum Methods Phys Res Sect A Accel Spectrom Detect Assoc Equip. https://doi.org/10.1016/j.nima.2009.10.059

8. Kudryavtsev VA, Spooner NJC, McMillan JE (2003) Simulations of muon-induced neutron flux at large depths underground. Nucl Instrum Methods Phys Res Sect A Accel Spectrom Detect Assoc Equip. https://doi.org/10.1016/S0168-9002(03)00983-5

9. UM5960 - CoMPASS User Manual (2019) CAEN, Access 17.05.2019

10. Mietelski JW, Hajduk Z, Hajduk L, Jurkowski J (2004) Background effects observed with a low-level gamma-spectrometer with muon veto detector. Conference \& Symposium Papers 26/P. In: International conference on isotopes in environmental studies, aquatic forum 2004, Monaco, 25-29 Oct. 2004, IAEACN-118/159, pp 538-542

11. Núñez-Lagos R, Virto A (1996) Shielding and background reduction. Appl Radiat Isot. https://doi.org/10.1016/S0969 -8043(96)00100-5

12. General Purpose Plastic Scintillator EJ-200, EJ-204, EJ-208, EH-212. https://eljentechnology.com/images/products/data_sheet s/EJ-200_EJ-204_EJ-208_EJ-212.pdf. Accessed 17 Sept 2019

13. UM3148 - DT5730/DT5725-User Manual (2016) CAEN, Access 20.05.2019

14. Jordanov VT, Knoll GF (1994) Digital synthesis of pulse shapes in real time for high resolution radiation spectroscopy. Nucl Instrum Methods Phys Res Sect A Accel Spectrom Detect Assoc Equip. https://doi.org/10.1016/0168-9002(94)91011-1

15. Guzik Z, Krakowski T (2013) Algorithms for digital $\gamma$-ray spectroscopy. Nukleonika 58(2):333-338

16. wxWidgets $3 \mathrm{C}++$ library, http://www.wxwidgets.org/. Accessed 17 Sept 2019

17. Application Note AN2508, CAEN digital pulse height analysera digital approach to radiation spectroscopy (2011) CAEN. Accessed 05 June 2019

18. Zhang W, Ro H, Liu C, Hoffman I, Ungar K (2017) Design and optimization of a dual-HPGe Gamma spectrometer and its cosmic veto system. IEEE Trans Nucl Sci. https://doi.org/10.1109/ TNS.2017.2654686
19. Paepen J, Schulte F, Mastinu P, Pedersen B, Saare H, Schillebeeckx P, Tkaczyk A, Varasano G (2016) Characterisation of plastic scintillators used as an active background shield for neutron detection. JRC Tech Rep. https://doi.org/10.2787/810820

20. Tkaczyk AH, Saare H, Ipbüker C, Schulte F, Mastinu P, Paepen J, Pedersen B, Schillebeeckx P, Varasano G (2018) Characterization of EJ-200 plastic scintillators as active background shield for cosmogenic radiation. Nucl Instrum Methods Phys Res Sect A Accel Spectrom Detect Assoc Equip. https://doi.org/10.1016/j. nima.2017.10.088

21. Yau C, Ho E (2019) Cosmic ray muon detection using NaI detectors and plastic scintillators. http://home.fnal.gov/ group/WORK/ muonDetection.pdf. Accessed 20 Mar 2019

22. Burnett JL, Davies AV (2014) Cosmic veto gamma-spectrometry for comprehensive nuclear-test-ban treaty samples. Nucl Instrum Methods Phys Res Sect A Accel Spectrom Detect Assoc Equip. https://doi.org/10.1016/j.nima.2014.02.027

23. Bossew P (2005) A very long-term HPGe-background gamma spectrum. Appl Radiat Isot. https://doi.org/10.1016/j.aprad iso.2004.09.006

24. Certified Reference Material IAEA-444 Gamma emitting radionuclides in soil, IAEA Environmental Laboratories, Date of issue: April 2010, Vienna, Austria

25. Reference Material IAEA-375, Radionuclides and trace elements in soil, Date of issue: January 2000, Vienna, Austria

26. Certified Reference Material IAEA-330, Radionuclides in spinach, Date of issue: May 2009, Vienna, Austria

27. Pittauer D, Höweling D, Pérez Mayo M, Hettwig B, Fischer HW (2016) SedActiv 1.0: a cosmic veto gamma spectrometry setup for sediment samples. In: 2nd international conference on radioecological concentration processes, Nov. 2016, Seville, Spain

28. Laubenstein M, Hult M, Gasparro J, Arnold D, Neumaier S, Heusserd G, Kohler M, Povinec P, Reyss JL, Schwaiger M, Theodorsson P (2004) Underground measurements of radioactivity. Appl Radiat Isot 61:167-172. https://doi.org/10.1016/j.aprad iso.2004.03.039

Publisher's Note Springer Nature remains neutral with regard to jurisdictional claims in published maps and institutional affiliations. 